\title{
Short range atomic migration in amorphous silicon
}

\author{
F. Strauß, ${ }^{1, a)}$ B. Jerliu, ${ }^{1}$ T. Geue, ${ }^{2}$ J. Stahn, ${ }^{2}$ and H. Schmidtt ${ }^{1,3}$ \\ ${ }^{1}$ AG Mikrokinetik, Institut für Metallurgie, TU Clausthal, D-38678 Clausthal-Zellerfeld, Germany \\ ${ }^{2}$ Laboratory for Neutron Scattering and Imaging, Paul Scherrer Institute, Villigen, CH-5232, Switzerland \\ ${ }^{3}$ Clausthaler Zentrum für Materialtechnik, TU Clausthal, D-38678 Clausthal-Zellerfeld, Germany
}

(Received 25 January 2016; accepted 16 April 2016; published online 2 May 2016)

\begin{abstract}
Experiments on self-diffusion in amorphous silicon between 400 and $500{ }^{\circ} \mathrm{C}$ are presented, which were carried out by neutron reflectometry in combination with ${ }^{29} \mathrm{Si} /{ }^{\text {nat }} \mathrm{Si}$ isotope multilayers. Short range diffusion is detected on a length scale of about $2 \mathrm{~nm}$, while long range diffusion is absent. Diffusivities are in the order of $10^{-19}-10^{-20} \mathrm{~m}^{2} / \mathrm{s}$ and decrease with increasing annealing time, reaching an undetectable low value for long annealing times. This behavior is strongly correlated to structural relaxation and can be explained as a result of point defect annihilation. Diffusivities for short annealing times of $60 \mathrm{~s}$ follow the Arrhenius law with an activation enthalpy of $(0.74 \pm 0.21) \mathrm{eV}$, which is interpreted as the activation enthalpy of Si migration. Published by AIP Publishing.

[http://dx.doi.org/10.1063/1.4948333]
\end{abstract}

\section{INTRODUCTION}

Amorphous silicon (a-Si) is a semiconductor with interesting properties for applications such as solar-cells, ${ }^{1,2}$ thin film transistors, ${ }^{3,4}$ Li-ion batteries, ${ }^{5}$ liquid crystal displays, ${ }^{6}$ image sensors, ${ }^{6}$ and light emission diodes. ${ }^{7}$ In addition, it is used as a precursor for the tailored production of nanocrystalline silicon. ${ }^{8}$ From a structural viewpoint, a-Si is a non-crystalline form of crystalline silicon. Ideally, it can be described as a covalently bound fourfold coordinated continuous network of silicon atoms ${ }^{8,9}$ and it is a perfect model system for an amorphous semiconductor. While short range order is similar for crystalline and amorphous Si modifications, bond length and bond angles deviate in a-Si from that in long range ordered $\mathrm{c}-\mathrm{Si}$ in a statistical way. ${ }^{10,11} \mathrm{In} \mathrm{a}-\mathrm{Si}$ about $20 \%$ fivefold coordinated $\mathrm{Si}$ is found compared with the fourfold coordination in crystalline $\mathrm{Si}^{12}$ Consequently, several dangling bonds are formed as defects, which are possible recombination centers for charge carriers. ${ }^{13}$ Thus, for applications passivation with hydrogen is commonly applied. ${ }^{14,15}$ However, the samples used in this study were deposited without the addition of hydrogen. Hence, the presented findings are not for hydrogenated amorphous silicon (a-Si:H) but specifically for hydrogen-free a-Si, yet they provide a base for further research even if the effects of hydrogen addition are drastic. Note that for both types of material (a-Si and a-Si:H) strongly different diffusivities and diffusion mechanisms may occur.

Atomic mobility of silicon atoms and consequently self-diffusion plays a very important role in a-Si because these quantities determine the intrinsic thermal stability. After synthesis by methods like chemical vapor deposition, magnetron sputtering, or ion-implantation of single crystals, amorphous silicon is in a non-equilibrium state. Annealing at elevated temperatures (e.g., for a modification of physical properties) results in a thermally activated

\footnotetext{
a) Author to whom correspondence should be addressed. Electronic mail: strauss.florian@tu-clausthal.de.
}

structural re-organization process which is termed structural relaxation. ${ }^{16,17}$ Structural relaxation refers to slow and short range atomic re-arrangement processes, which lead to a reduction in free energy while retaining the amorphous phase. ${ }^{18}$ This re-structuring of the amorphous network leads to an increase of short range order and is described as arising from the superposition of elementary processes with varying activation energies. ${ }^{19}$ Structural relaxation can also be interpreted in the framework of point defect annihilation processes. ${ }^{16,20,21}$ Defect densities and consequently application relevant optical and electronic properties may be influenced in a direct way. ${ }^{13}$ As further described in Ref. 22 , an exact modeling and simulation of the crystallization behavior of a-Si is possible only with the knowledge of self-diffusivities.

Although silicon single crystals are one of the best characterized types of materials, also concerning their diffusion properties, ${ }^{23,24}$ there actually exist no reliable experimental data on self-diffusivities in a-Si except our own previous work. $^{25,26}$ This fact results from the combination of the intrinsic structural metastability of a-Si in combination with the expected low diffusivities due to covalent bonding. Recently, we published results on self-diffusion in amorphous silicon thin films in the temperature range between 550 and $700{ }^{\circ} \mathrm{C}$. The diffusivities obey the Arrhenius law with an activation enthalpy of $4.4 \mathrm{eV}$, which is only $0.55 \mathrm{eV}$ lower than that found for crystalline silicon at temperatures above $900{ }^{\circ} \mathrm{C} .{ }^{26}$ The activation enthalpy is assumed to be composed of a formation and a migration part of (essentially unknown) defects that govern diffusion. ${ }^{26}$ No time dependence of diffusivities could be proven in the investigated time-temperature domain above $500{ }^{\circ} \mathrm{C} .{ }^{25,26}$ At about $700{ }^{\circ} \mathrm{C}$ the diffusivities of a-Si are about five orders of magnitude higher than that in crystalline silicon (c-Si), which is traced back to an anomalous high pre-exponential factor and hence diffusion entropy.

In the present paper we report on measurements of selfdiffusion in amorphous silicon in the low temperature range 


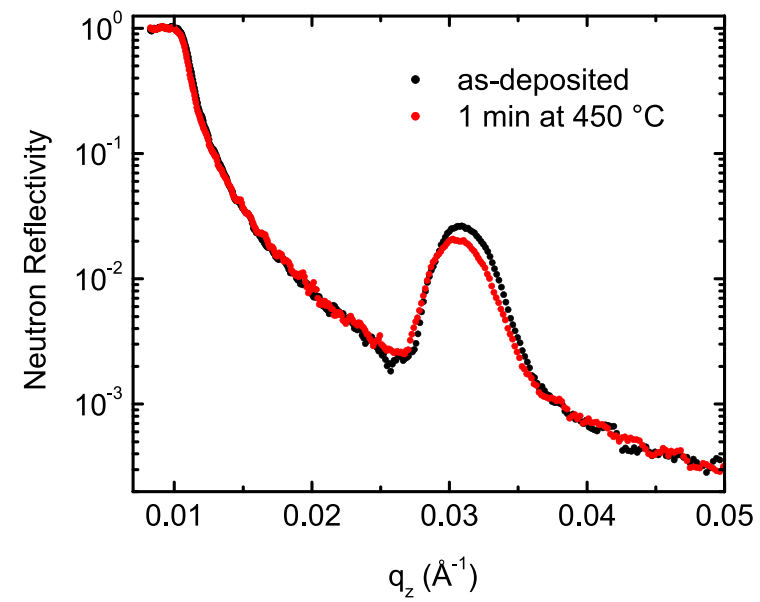

FIG. 1. Neutron reflectivity patterns of amorphous $\left[{ }^{29} \mathrm{Si} /{ }^{\text {nat }} \mathrm{Si}\right]_{10}$ isotope multilayers in the as-deposited state and after annealing at $450^{\circ} \mathrm{C}$ for $1 \mathrm{~min}$.

between 400 and $500^{\circ} \mathrm{C}$. The experiments are done by neutron reflectometry (NR) in combination with ${ }^{29} \mathrm{Si} /{ }^{\text {nat }} \mathrm{Si}$ isotope multilayers. This method allows measuring low diffusivities down to $10^{-25} \mathrm{~m}^{2} / \mathrm{s}$ and very small diffusion lengths of $1 \mathrm{~nm}$ and below. ${ }^{24,27,28}$ Consequently, it is perfectly suited to study silicon self-diffusion at low temperatures and on small length scales.

\section{EXPERIMENTAL}

Isotope multilayers are stacks of several nanometer thick double layers, with each single layer enriched with a different stable isotope of a certain element. In the present case, $\left[{ }^{29} \mathrm{Si}(5 \mathrm{~nm}) /{ }^{\text {nat }} \mathrm{Si}(16 \mathrm{~nm})\right] \times 10$ structures $\left({ }^{\text {nat }} \mathrm{Si}\right.$ contains $92.2 \%$ of ${ }^{28} \mathrm{Si}$ ) were deposited by ion-beam sputtering on (100) oriented Si wafers. For sputtering, a commercial ion beam sputter unit (IBC 681, Gatan) with two Penning ion guns was used. The base pressure of the vacuum chamber was $5 \times 10^{-7}$ mbar. Sputtering was done with Ar ions at a working pressure of $5 \times 10^{-5}$ mbar. The ion beam acceleration voltage was $5 \mathrm{kV}$ and the ion beam current was $180 \mu \mathrm{A}$. During deposition, the specimen is rotated and rocked to ensure a uniform coating of the sample. Different target materials can be selected during sputtering without breaking the vacuum. As ${ }^{\text {nat }} \mathrm{Si}$ sputter target a commercial, nominally undoped $\mathrm{Si}$ wafer was used, while the ${ }^{29} \mathrm{Si}$ target was synthesized by melting commercially available ${ }^{29} \mathrm{Si}$ silicon flakes (enrichment: $99.34 \%$ ) from Isoflex, USA, in a boron nitride crucible at $1405^{\circ} \mathrm{C}$ in vacuum better than $10^{-5}$ mbar. Afterward, the target was ground and polished in order to remove surface contaminations. Prior to multilayer deposition, each target was pre-sputtered for $15 \mathrm{~min}$ to remove a possible additional contaminated surface layer. The resulting sputtered multilayers contain a maximum content of $5 \%$ carbon, which is located in the ${ }^{29} \mathrm{Si}$ layers. ${ }^{25}$ This enhanced carbon concentration results from contamination of the source powder material of the target as provided by the supplier. During annealing, carbon remains located in the ${ }^{29} \mathrm{Si}$ layer and is not influencing the diffusion process. ${ }^{25}$

All samples discussed in this study are X-ray amorphous as proven by grazing incidence diffractometry (GI-XRD), using a Bruker D 5000 machine. $^{25,26}$ The isotope modulation of the samples in the direction perpendicular to the surface was verified by Secondary Ion Mass Spectrometry (SIMS). Due to the difference in neutron scattering lengths of ${ }^{29} \mathrm{Si}$ $(4.70 \mathrm{fm})$ and ${ }^{\text {nat }} \mathrm{Si}(4.15 \mathrm{fm})$ this system is well-suited for neutron scattering. Neutron reflectometry experiments were carried out at the Swiss spallation neutron source (SINQ, PSI Villigen). The time-of-flight reflectometer AMOR was used, and reflectivity patterns were measured at incident angles between $0.3^{\circ}$ and $1.3^{\circ}$, respectively, after each annealing period and cooling down to room temperature. The resolution of the reflectometer was about $\Delta \mathrm{q}_{\mathrm{z}} / \mathrm{q}_{\mathrm{z}}=5 \%$.

Diffusion annealing of the multilayers was done in a rapid thermal annealing system (AO 500, MBE) located at the neutron facility, where the samples are annealed in argon at ambient pressure. The desired temperature is achieved in about $10 \mathrm{~s}$ after the start of the annealing procedure. Additional long-term anneals were carried out in a homemade resistive annealing set-up in a silica tube in argon.

\section{RESULTS AND DISCUSSION}

Characteristic neutron reflectometry patterns as recorded during the experiments are shown in Fig. 1 exemplarily for an as-deposited sample and a sample annealed at $450{ }^{\circ} \mathrm{C}$ for $1 \mathrm{~min}$. A clear Bragg peak is visible at a wave vector of $0.031 \AA^{-1}$, resulting from the reflection of the neutrons at the isotope interfaces and the corresponding interference effects. Isothermal annealing of the isotope multilayer leads to interdiffusion of the two isotopes. This results in a decrease of the Bragg peak, as also shown in Fig. 1. For further analysis, first the background beyond the Bragg peaks resulting from Fresnel reflectivity is subtracted and afterward the peak is
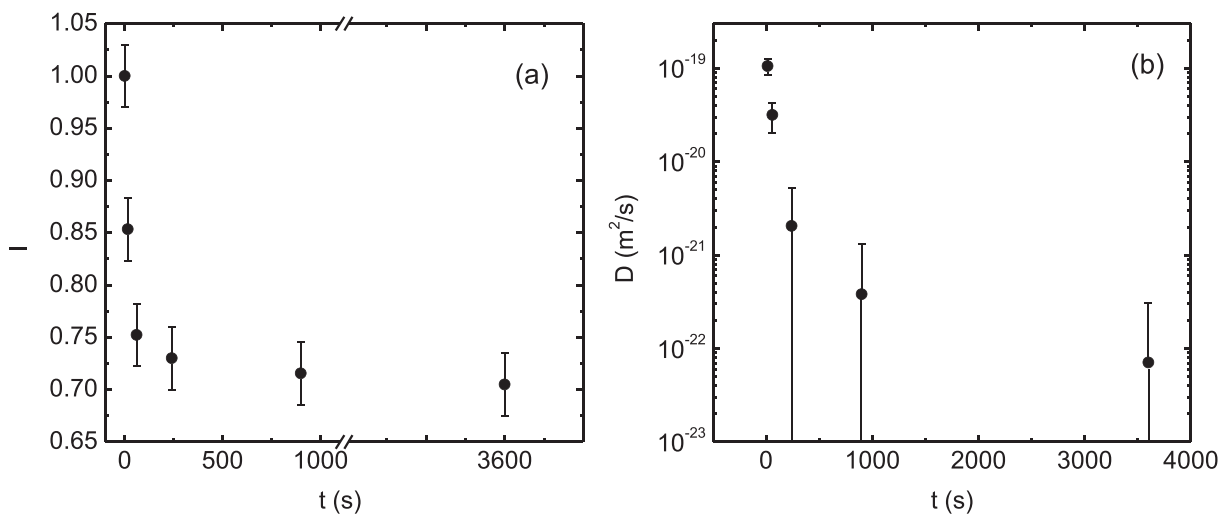

FIG. 2. (a) Integrated intensity of the Bragg peak and (b) consecutive diffusivities as a function of annealing time at $450{ }^{\circ} \mathrm{C}$. 
fitted by a Gaussian function from which the integrated intensity of the Bragg peak, $I$, is derived.

In Fig. 2(a) the integrated intensity of the area under the Bragg peak, $I$, normalized to its initial value, $I_{0}$, is plotted as a function of annealing time between $15 \mathrm{~s}$ and $1 \mathrm{~h}$ for a temperature of $450{ }^{\circ} \mathrm{C}$. Similar measurements were done at 400 and $500{ }^{\circ} \mathrm{C}$, respectively. We observe a continuous decrease of the peak during the first $60 \mathrm{~s}$ of annealing. Afterward the intensity decrease slows down and a constant value of about $70 \%$ of the initial value is reached. This behavior can be explained with the occurrence of experimentally detectable diffusion of silicon atoms in the initial stages of the annealing process and an "immobilization" of the atoms afterward. Also prolonged annealing of a sample up to $109 \mathrm{~h}$ at $500^{\circ} \mathrm{C}$ does not lead to a further significant intensity decrease. Annealing at temperatures of $550^{\circ} \mathrm{C}$ and above, where long range diffusion is present, $^{25,26}$ clearly shows that a further reduction of the Bragg peak to values lower than $70 \%$ of the initial value can be observed. This demonstrates that diffusion is frozen-in for long annealing times at the given temperature and atomic motion is restricted to short annealing times and consequently to short range distances of 1 to $3 \mathrm{~nm}$ (as will be shown later).

From the modification of the Bragg peak intensity the actual self-diffusivities can be calculated according to the $\operatorname{expression}^{29}$

$$
D=-\ln \left(\frac{I_{i+1}-I_{e}}{I_{i}-I_{e}}\right) \frac{d^{2}}{8 \pi^{2} \Delta t}
$$

where $I_{\mathrm{i}}$ and $I_{\mathrm{i}+1}$ are the intensities of the Bragg peak after annealing for times $t_{i}$ and $t_{i+1}$, respectively, $I_{\mathrm{e}}=0.4$ is the saturation intensity after high temperature annealing as found in Ref. $26, d$ is the bi-layer thickness of a ${ }^{29} \mathrm{Si} /{ }^{\mathrm{nat}} \mathrm{Si}$ unit, and $\Delta t$ is the difference in annealing time between annealing step $\mathrm{i}+1$ and $\mathrm{i}$. The results at $450{ }^{\circ} \mathrm{C}$ are given in Fig. 2(b). As obvious, diffusivities with error limits of less than $100 \%$ can be calculated for annealing times of $15 \mathrm{~s}$ and 60 s only. For higher annealing times, the difference between consecutive intensity values is too small to obtain exact diffusivities. Due to the high error limits, only an upper limit can be given. The data in Fig. 2(b) (exact diffusivities and upper limits) clearly confirm the suggested diffusivity decrease as a function of annealing time. From the fact that $109 \mathrm{~h}$ of annealing at $500{ }^{\circ} \mathrm{C}$ (not shown) does not lead to a measurable additional Bragg peak decrease, the upper limit of diffusivities at this temperature (and below) is assessed to be less than $1 \times 10^{-24} \mathrm{~m}^{2} / \mathrm{s}$.

The observed decrease of the diffusivities during annealing (see Fig. 2(b)) can be explained as the result of a defect annihilation process. We generally assume that self-diffusion in silicon is governed by a defect-atom exchange process, where $D_{\mathrm{Si}}=c_{\mathrm{D}} D_{\mathrm{D}}$ is valid. Here, $D_{\mathrm{Si}}$ is the diffusivity of the $\mathrm{Si}$ atoms, $c_{\mathrm{D}}$ is the atomic fraction of the (not specified) defect species governing diffusion, and $D_{\mathrm{D}}$ is the diffusivity of the defects. We now assume that at the beginning of the annealing process a large number of non-equilibrium defects is present in excess of the thermal equilibrium value. Consequently, diffusion is governed by these excess defects. During annealing the number of available defects and consequently the diffusivities are reduced, leading to this transient effect. This reduction of defects might be due to annihilation at sinks taking place during structural relaxation. A classic example is the annihilation of vacancies by interstitials but also the reduction of the number of other defects like dangling bonds or simply the reduction of free volume. Due to the fact that the diffusivity is directly proportional to the defect concentration, the behavior of diffusivities maps the behavior of defects. The occurrence of constant intensity values in Fig. 2(a) for long annealing times means that a final constant concentration of diffusion defects is established. At this point structural relaxation (and correlated defect annihilation) is no longer operating and only thermal defects are present for controlling the diffusion process. Diffusivities (and the concentration of diffusion defects) are now so low that a significant modification cannot be observed during annealing within reliable times (several days) for temperatures below $500^{\circ} \mathrm{C}$. As shown in Ref. 26, the situation is different for temperatures between 550 and $700^{\circ} \mathrm{C}$. There, defect annihilation is immeasurably fast, but diffusion in thermal equilibrium can be probed. Consequently, at these high temperatures no time dependence of diffusivities is observed. ${ }^{26}$

A limited diffusion range of silicon atoms might also occur if a broad distribution of hopping barriers is present (due to structural disorder) that have to be crossed during diffusion. Such an arrangement might contribute to the observed limitation of diffusion to a length scale of some few nanometers. The atoms may only move in a given spatial range distinguished by low barriers and cannot leave a cage-like area formed by higher barriers. Atomistic calculations recently found evidence for such a hopping barrier distribution. ${ }^{30}$

In order to get more insight into the diffusion behavior at short annealing times, the diffusivities calculated from the intensity difference between the as-deposited sample and the sample annealed for $1 \mathrm{~min}$ at 400,450 , and $500{ }^{\circ} \mathrm{C}$ as a

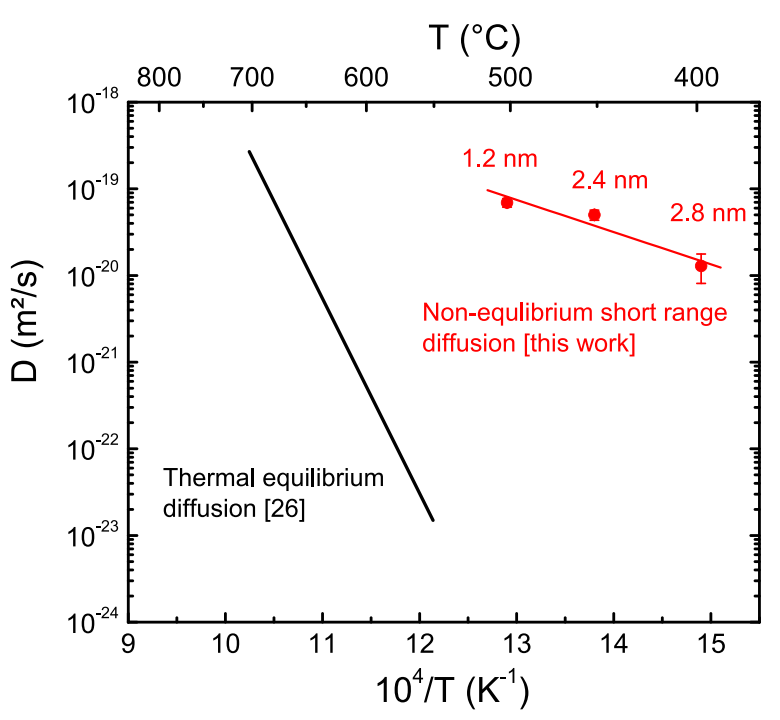

FIG. 3. Self-diffusivities in amorphous silicon as a function of reciprocal temperature at low temperatures between 400 and $500^{\circ} \mathrm{C}$ in comparison with literature high temperature data above $500^{\circ} \mathrm{C}$ as given in our previous work. ${ }^{26}$ Diffusivities of the present work were calculated for the difference in Bragg peak intensity between the as-deposited sample and the sample annealed for $60 \mathrm{~s}$. The corresponding diffusion length for each diffusivity value is indicated. 
function of reciprocal temperature are plotted in Fig. 3. The annealing time period of $60 \mathrm{~s}$ was chosen because data are available for all temperatures investigated, which is not the case for $15 \mathrm{~s}$. The diffusion lengths $d=(2 D t)^{1 / 2}$ connected with the diffusivities are also indicated in Fig. 3. As obvious, values less than $3 \mathrm{~nm}$ are found, which means that short range atomic hopping of only some atomic jumps takes place. Such small diffusion lengths can exclusively be detected by NR and are hardly measurable by classical (e.g., SIMS based) methods which probe diffusion generally only for diffusion lengths of several tens of nanometers. If at $500{ }^{\circ} \mathrm{C}$ the diffusion annealing is prolonged to $109 \mathrm{~h}$ the additional diffusion length of the atoms during this time period is below $0.5 \mathrm{~nm}$ due to the decreased diffusivity and consequently not detectable. As an important result we find that the atomic migration of silicon as detectable by NR in the temperature range between 400 and $500{ }^{\circ} \mathrm{C}$ is limited to short ranges of some nanometers. We expect that this short range migration is strongly correlated to atomic rearrangement taking place during structural relaxation.

A least-square fit of the data set given in Fig. 3, corresponding to diffusivities of $60 \mathrm{~s}$, to the Arrhenius equation

$$
D=D_{0} \exp \left(-\Delta \mathrm{H}_{\mathrm{D}} / \mathrm{k}_{\mathrm{B}} \mathrm{T}\right)
$$

results in an activation enthalpy of diffusion of $\Delta H_{D}$ $=(0.74 \pm 0.21) \mathrm{eV}$ and a pre-exponential factor of $D_{0}=5$ $\times 10^{-15} \mathrm{~m}^{2} / \mathrm{s}$ (error: $\left.\ln \left(D_{0} / \mathrm{m}^{2} \mathrm{~s}^{-1}\right)=3.4\right)$, respectively. The activation enthalpy of diffusion is rather low.

Also shown in Fig. 3 are recent literature data on silicon self-diffusivities in amorphous silicon at temperatures above $500{ }^{\circ} \mathrm{C},{ }^{26}$ which follow an activation enthalpy of $4.4 \mathrm{eV}$ and which are correlated to thermal equilibrium (long range) diffusion. The diffusivities found in the high temperature range are significantly lower than those found in the present experiments if extrapolated to the temperature range between 400 and $500{ }^{\circ} \mathrm{C}$. For example, a diffusivity of $1 \times 10^{-25} \mathrm{~m}^{2} / \mathrm{s}$ at $500{ }^{\circ} \mathrm{C}$ is extrapolated from the data of Ref. 26 , about one order of magnitude lower than the upper limit of $1 \times 10^{-24} \mathrm{~m}^{2} / \mathrm{s}$ found in this study. The high activation enthalpy of $4.4 \mathrm{eV}$ emphasizes that at high temperatures thermal equilibrium defects and consequently the defect formation enthalpy, $\Delta H_{D}{ }^{\mathrm{f}}$, are involved.

The explanation for the low activation enthalpy of about $0.7 \mathrm{eV}$ found in the low temperatures range is the following: During deposition defects are formed in the a-Si network. Thus, at the beginning of the annealing process the amount of diffusion defects is relatively high, much higher than the number of equilibrium defects present at the end of the relaxation process. Consequently, the activation enthalpy can be identified with the defect migration enthalpy $\left(\Delta H_{D}=\Delta H_{D}{ }^{\mathrm{m}}\right)$. As mentioned, at higher temperatures between 550 and $700{ }^{\circ} \mathrm{C}$ the sum of the migration and the formation enthalpy is measured. ${ }^{26}$

The idea of diffusion that is governed by nonequilibrium defects in the present case is also supported by the size of the pre-exponential factor. In contrast to the factor of thermal equilibrium diffusion at high temperatures, which is rather high $\left(D_{0}=1.5 \times 10^{4}\right)$, in the present case it is about 20 orders of magnitude lower $\left(D_{0}=5 \times 10^{-15} \mathrm{~m}^{2} / \mathrm{s}\right)$.
The pre-exponential factor is given by

$$
D_{0}=a^{2} \nu_{0} \exp \left(\Delta S / k_{\mathrm{B}}\right)
$$

for thermal defects and

$$
D_{0}=f_{\mathrm{v}} a^{2} \nu_{0} \exp \left(\Delta S_{m} / k_{\mathrm{B}}\right),
$$

for structural defects, neglecting the correlation factors. Here, $a=2.35 \AA$ is the $\mathrm{Si}-\mathrm{Si}$ atomic distance, ${ }^{31} \nu_{0} \approx 1.3 \times 10^{13} \mathrm{~s}^{-1}$ is the Debye frequency, ${ }^{31} f_{\mathrm{v}}$ is the mole fraction of structural defects, $\Delta S_{m}$ is the migration part of the entropy of diffusion, and $\Delta S$ is the sum of the migration part and the defect formation part. Applying Equation (3) to our low pre-exponential factor allows us to calculate a strong negative entropy of diffusion to $\Delta S_{m}=-19 \mathrm{k}_{B}$, which is not reasonable. This again confirms diffusion via non-equilibrium structural defects. Consequently, we can use Equation (4) and assume that $\Delta S_{m}$ is ranging between 1 and $10 \mathrm{k}_{\mathrm{B}}$, as the mole fraction of defects, $f_{\mathrm{v}}$, between $10^{-9}$ and $10^{-13}$ is assessed from Equation (4). In conclusion, using NR at temperatures above $500^{\circ} \mathrm{C}$, diffusion in thermal equilibrium can be proved, while at temperatures of $500{ }^{\circ} \mathrm{C}$ and below, diffusion via "structural nonequilibrium defects" is detected.

Fig. 3 will also supply information why at high temperatures $^{26}$ no time-dependent diffusivities are observed due to structural relaxation processes. If the Arrhenius straight line in Fig. 3 is extrapolated to higher temperatures it will intersect the diffusivities in the high temperature range around $700{ }^{\circ} \mathrm{C}$. That means that around this temperature transient diffusion effects at very short annealing times are weakly pronounced because the diffusivities of both processes (via thermal and structural defects) are roughly identical and cannot be distinguished.

In literature, a theoretical study on self-diffusion in amorphous silicon can be found which was done between 627 and $1027^{\circ} \mathrm{C}$ by classical molecular dynamics. ${ }^{12}$ An activation enthalpy of silicon migration of about $0.9 \mathrm{eV}$ is derived $^{12}$ which is in good accordance to our results. However, the absolute diffusivities calculated in that study are several orders of magnitude too high if extrapolated to, e.g., $400^{\circ} \mathrm{C}$. In a different study an activation enthalpy of only $0.23 \mathrm{eV}$ is calculated at low temperatures between 27 and $327^{\circ} \mathrm{C}$ (Ref. 22) in contradiction to our results. In Ref. 12 , as a possible diffusion mechanism, atomic rearrangement processes like bond break (dominating) or bond switch as well as interstitial-like configurations are suggested. Other work gives dangling bonds, ${ }^{32}$ floating bonds, ${ }^{33}$ or vacancy-like defects ${ }^{34}$ as the dominating defects. Defects with a broad spectrum of activation energies between 0.23 and $2.7 \mathrm{eV}$ are also suggested, indicating a multiplicity of mechanisms. $^{35}$

In Ref. 26, where the temperature range above $500{ }^{\circ} \mathrm{C}$ was explored, diffusion via extended interstitial defects (based on Ref. 36) was postulated in order to explain the high pre-exponential factor by a high entropy of diffusion. However, it is also stated in Ref. 36 that at low temperatures conventional self-interstitial defects are expected to be present. Further, the structural non-equilibrium defects governing 
diffusion below $500^{\circ} \mathrm{C}$ and the thermal defects governing diffusion above $500^{\circ} \mathrm{C}$ are also not necessarily the same. Consequently, the presence of extended defects as postulated in the high temperature range is not very likely in the low temperature range. From these considerations and literature data it is not possible to identify the short range diffusion mechanism at low temperatures in amorphous silicon.

For further insight, measurements on samples where dangling bonds are saturated by hydrogen are planned in the near future. There, the addition of hydrogen may produce a radically different self-diffusion behavior if diffusion is mediated by dangling bonds. As stated earlier, dangling bonds present in the as-deposited material will be saturated by hydrogen. This means that less of these defects are present in the material and diffusion is expected to slow down, leading to lower diffusivities at the same annealing time and temperature used in the present study. Additionally, if (as stated earlier) structural relaxation of the continuous silicon network is influenced by the number of dangling bonds, a hydrogen saturation of those bonds would lead to a less pronounced structural relaxation behavior and, consequently, to a reduced decrease of diffusivities over time.

\section{CONCLUSION}

In conclusion, during the present experiments selfdiffusion in nearly hydrogen-free amorphous silicon was probed by ${ }^{29} \mathrm{Si} /{ }^{\text {nat }} \mathrm{Si}$ isotope multilayers in combination with neutron reflectometry. The results show that at temperatures between 400 and $500{ }^{\circ} \mathrm{C}$ only short range diffusion on a length scale of about $2 \mathrm{~nm}$ can be observed. Time-dependent diffusivities are found which are explained by defect annihilation processes, probably correlated to structural relaxation. Diffusivities for short annealing times of $60 \mathrm{~s}$ follow the Arrhenius law, with a low activation enthalpy of about $0.7 \mathrm{eV}$ corresponding to the enthalpy of defect migration. Long range diffusion could not be detected to an upper limit of $1 \times 10^{-24} \mathrm{~m}^{2} / \mathrm{s}$ at $500^{\circ} \mathrm{C}$.

\section{ACKNOWLEDGMENTS}

This work is based on experiments performed on the reflectometer Amor at the Swiss spallation neutron source SINQ, Paul Scherrer Institute, Villigen, Switzerland. This research has been supported by the German Research Foundation under the Contract No. Schm1569/22-1 and by the European Commission under the 7th Framework Programme through the "Research Infrastructures" action of the "Capacities" Programme, NMI3-II Grant No. 283883.
${ }^{1}$ A. Shah, P. Torres, R. Tscharner, N. Wyrsch, and H. Keppner, Science 285, 692 (1999).

${ }^{2}$ M. Kondo, T. Matsui, Y. Nasuno, H. Sonobe, and S. Shimizu, Thin Solid Films 501, 243 (2006).

${ }^{3}$ M. J. Powell, IEEE Trans. Electron Devices 36, 2753 (1989).

${ }^{4} \mathrm{H}$. Gleskova and S. Wagner, IEEE Electron Device Lett. 20, 473 (1999).

${ }^{5}$ M. T. McDowell, S. W. Lee, W. D. Nix, and Y. Cui, Adv. Mater. 25, 4966 (2013).

${ }^{6}$ A. Madan, J. Non-Cryst. Solids 352, 881 (2006).

${ }^{7}$ P. Servati, S. Prakash, A. Nathan, and C. Py, J. Vac. Sci. Technol. A 20, 1374 (2002).

${ }^{8}$ C. Spinella, S. Lombardo, and F. Priolo, J. Appl. Phys. 84, 5383 (1998).

${ }^{9}$ D. E. Polk and D. S. Boudreaux, Phys. Rev. Lett. 31, 92 (1973).

${ }^{10}$ S. R. Elliott, Adv. Phys. 38, 1 (1989).

${ }^{11}$ S. Kugler and Z. Várallyay, Philos. Mag. Lett. 81, 569 (2001).

${ }^{12}$ I. Santos, L. A. Marqués, L. Pelaz, and L. Colombo, Phys. Rev. B 83, 153201 (2011).

${ }^{13}$ C. Boehme, F. Friedrich, T. Ehara, and K. Lips, Thin Solid Films 487, 132 (2005).

${ }^{14}$ R. A. Street, Phys. B: Condens. Matter 170, 69 (1991).

${ }^{15}$ W. Beyer and U. Zastrow, J. Non-Cryst. Solids 227-230, 880 (1998).

${ }^{16}$ S. Roorda, S. Doorn, W. C. Sinke, P. M. L. O. Scholte, and E. van Loenen, Phys. Rev. Lett. 62, 1880 (1989).

${ }^{17}$ I. Štich, R. Car, and M. Parrinello, Phys. Rev. B 44, 11092 (1991).

${ }^{18}$ J. H. Shin and H. A. Atwater, Phys. Rev. B 48, 5964 (1993).

${ }^{19}$ P. Roura and J. Farjas, Acta Mater. 57, 2098 (2009).

${ }^{20}$ S. Roorda, W. C. Sinke, J. M. Poate, D. C. Jacobson, S. Dierker, B. S. Dennis, D. J. Eaglesham, F. Spaepen, and P. Fuoss, Phys. Rev. B 44, 3702 (1991).

${ }^{21}$ S. Coffa, F. Priolo, and A. Battaglia, Phys. Rev. Lett. 70, 3756 (1993).

${ }^{22}$ L. Brambilla, L. Colombo, V. Rosato, and F. Cleri, Appl. Phys. Lett. 77, 2337 (2000).

${ }^{23} \mathrm{H}$. Bracht and N. A. Stolwijk, in Diffusion in Semiconductors, edited by D. Beke (Springer, Berlin, Heidelberg, 1998), pp. 1-11.

${ }^{24}$ R. Kube, H. Bracht, E. Hüger, H. Schmidt, J. L. Hansen, A. N. Larsen, J. W. Ager, E. E. Haller, T. Geue, and J. Stahn, Phys. Rev. B 88, 85206 (2013).

${ }^{25}$ F. Strauß, T. Geue, J. Stahn, and H. Schmidt, Defect Diffus. Forum 363, 225 (2015).

${ }^{26}$ F. Strauß, L. Dörrer, T. Geue, J. Stahn, A. Koutsioubas, S. Mattauch, and H. Schmidt, Phys. Rev. Lett. 116, 25901 (2016).

${ }^{27}$ H. Schmidt, M. Gupta, and M. Bruns, Phys. Rev. Lett. 96, 55901 (2006).

${ }^{28}$ E. Hueger, U. Tietze, D. Lott, H. Bracht, D. Bougeard, E. E. Haller, and H. Schmidt, Appl. Phys. Lett. 93, 162104 (2008).

${ }^{29}$ S. Chakravarty, H. Schmidt, U. Tietze, D. Lott, N. Lalla, and A. Gupta, Phys. Rev. B 80, 14111 (2009).

${ }^{30}$ G. A. Tritsaris, K. Zhao, O. U. Okeke, and E. Kaxiras, J. Phys. Chem. C 116, 22212 (2012).

${ }^{31}$ G. Kissinger and S. Pizzini, Silicon, Germanium, and Their Alloys (Taylor and Francis, Hoboken, 2014).

${ }^{32}$ W. G. Spitzer, G. K. Hubler, and T. A. Kennedy, Nucl. Instrum. Methods Phys. Res. 209-210, 309 (1983).

${ }^{33}$ S. T. Pantelides, Phys. Rev. Lett. 58, 1344 (1987).

${ }^{34}$ G. N. van den Hoven, Z. N. Liang, L. Niesen, and J. S. Custer, Phys. Rev. Lett. 68, 3714 (1992).

${ }^{35}$ G. T. Barkema, Phys. Rev. Lett. 81, 1865 (1998).

${ }^{36}$ N. E. B. Cowern, S. Simdyankin, C. Ahn, N. S. Bennett, J. P. Goss, J.-M. Hartmann, A. Pakfar, S. Hamm, J. Valentin, E. Napolitani, D. de Salvador, E. Bruno, and S. Mirabella, Phys. Rev. Lett. 110, 155501 (2013). 